\title{
PHYSICAL RESEARCH LABORATORY RADIOCARBON DATE LIST I
}

\author{
D P AGRAWAL, R V KRISHNAMURTHY, SHEELA KUSUMGAR, \\ and R K PANT
}

Physical Research Laboratory, Ahmedabad 380009, India

As the Radiocarbon Lab of the Tata Institute, Bombay, has now been shifted to the Physical Research Laboratory (PRL), Ahmedabad, our new date lists will be termed as above and the samples will be given PRL numbers instead of the former TF. All the pending TF samples were given new PRL numbers.

Presented below are dates from some important archaeologic sites. All dates are based on $\tau_{1 / 2}=5568 \mathrm{yr}$; to convert them to $\mathrm{AD} / \mathrm{BC}$ scale, 1950 has been used as the base year.

Samples were converted to methane for measuring ${ }^{14} \mathrm{C}$ activity in gas proportional counters. Detailed techniques were described earlier (R, 1971, v 13, p 442-449).

General Comment: a number of ${ }^{14} \mathrm{C}$ dates (PRL-81, -83, -67, -68) now confirm that the Painted Grey Ware culture extended up to the 3rd century BC. Some dates from the so-called Chalcolithic sites from Barkhera (PRL-113), Bhimbetka (PRL-17), and Koldihawa (PRL-100, -101) are too old. This may indicate some hitherto unknown basal cultures in these regions. ${ }^{14} \mathrm{C}$ dates on in situ Megalithic material do not seem to go back beyond 200 BC (PRL-134, -135).

\section{ACKNOWLEDGMENTS}

We thank M P K Kurup and K K Shivsankar for glass blowing help and N B Vaghela for laboratory assistance.

\section{SAMPLE DESGRIPTIONS}

ARCHAEOLOGIC SAMPLES

\section{Allahapur series, Uttar Pradesh}

Allahapur, Dist Meerut, subm by Dir Gen, Archaeol, New Delhi, from Painted Grey Ware (PGW) and Northern Black Polished Ware (NBP) levels. Samples date early Iron age.

PRL-81. PGW deposits

$2270 \pm 90$ 320 вC

Charcoal, Loc Tr A1, Qd 1, Layer 11, depth 2.3m, Field ALP/2/7071. $\mathrm{NaOH}$ pretreatment.

PRL-83. PGW-NBP overlap

$2160 \pm 105$

210 BC

Charcoal, Loc Tr A1, Qd 4, Layer 5, depth 1.1m, Field ALP/4/7071. $\mathrm{NaOH}$ pretreatment. 
PRL-66. Ambamata, India, old copper working area

$$
830 \pm 100
$$

Timber from Bore Hole 100 at depth $62 \mathrm{~m}$ near Ambamata $\left(24^{\circ} 20^{\prime}\right.$ N, $72^{\circ} 52^{\prime}$ E), Dist Banaskantha. Coll July 1971 by N C Shekar; subm by Dir, Geol Survey India, Ahmedabad. $\mathrm{NaOH}$ pretreatment.

\section{Barkhera series, Madhya Pradesh}

Barkhera $\left(22^{\circ} 9^{\prime} \mathrm{N}, 77^{\circ} 7^{\prime} \mathrm{E}\right)$, Dist Raisen, subm by $\mathrm{K} \mathrm{D}$ Bajpai, Sagar Univ, Sagar.

\section{PRL-111. Chalcolithic culture}

$3170 \pm 105$

Charcoal, Layers II and III, depth .1 to .2m, Field C-26. NaOH pretreatment.

\section{PRL-113. Chalcolithic culture}

$$
7250 \pm 135
$$

Seeds, Layers II and III, depth .1 to .2m, Field C-26. NaOH pretreatment. Comment: obviously these are older, perhaps wild seeds and have no relevance to Chalcolithic levels.

\section{PRL-15. Bharatpur, India, Chalcolithic-}

\section{Neolithic culture}

$$
\begin{aligned}
& 3290 \pm 135 \\
& 1340 \mathrm{BC}
\end{aligned}
$$

Charcoal from Bharatpur $\left(23^{\circ} 24^{\prime} \mathrm{N}, 87^{\circ} 27^{\prime} \mathrm{E}\right)$, Dist Burdwan, Loc Tr G5-H5, Layer 5, depth 1m; subm by Dir Gen, Archaeol, New Delhi. $\mathrm{NaOH}$ pretreatment.

\section{Bhimbetka series, Madhya Pradesh}

Bhimbetka (22。 $57^{\prime} \mathrm{N}$, $\left.77^{\circ} 37^{\prime} \mathrm{E}\right)$, Dist Raisen; subm by $\mathrm{V} \mathrm{S}$ Wakankar, Vikram Univ, Ujjain. Comment: dates show wide scatter; younger dates probably represent later disturbances at the site.

PRL-49. Burial 1

\section{Modern}

Charcoal from lower part of skeleton, Loc Tr III F-16, Field 5. Rootlets removed, $\mathrm{NaOH}$ pretreatment.

\section{PRL-50. Burial 3}

Charcoal from pit, Loc Tr III F-13, depth .03 to .1m, Field 6. Rootlets removed, $\mathrm{NaOH}$ pretreatment.

\section{PRL-51. Burial 2}

$$
2050 \pm 110
$$

Charcoal from pit, Loc Tr III F-13, Field 7. Rootlets removed, $\mathrm{NaOH}$ pretreatment.

\section{PRL-17. Pit deposit}

$7570 \pm 210$

Charcoal, Pit 3, depth .6m, NaOH pretreatment. 
PRL-18. Pit deposit

$2650 \pm 125$

Charcoal, Pit 11, depth 2.76m.

$700 \mathrm{BC}$

PRL-108. Henagahapugala, Ceylon, Coastal deposit 1010 вс

Shells from emerged beach lagoon, indirectly assoc with Mesolithic artifacts near Henagahapugala $\left(6^{\circ} 4^{\prime} 55^{\prime \prime} \mathrm{N}, 80^{\circ} 55^{\prime} 50^{\prime \prime} \mathrm{E}\right)$, Dist Hambantota, Loc G2, Layer II, depth 8m; subm by Dir Archaeol, Ceylon.

\section{Inamgaon series Maharashtra}

Inamgaon $\left(18^{\circ} 25^{\prime} \mathrm{N}, 74^{\circ} 32^{\prime} \mathrm{E}\right)$, Dist Poona, a Chalcolithic site with a sequence from Malwa to Late Jorwe cultures; subm by Dir, Deccan College, Poona.

PRL-57. Chalcolithic culture

$3050 \pm 105$

$1100 \mathrm{BC}$

Charcoal, Loc INM-I, Tr D8, Layer 3, depth .75m, Field INM-I/884. $\mathrm{NaOH}$ pretreatment.

PRL-59. Chalcolithic culture

$3210 \pm 110$

Charcoal, Loc INM-I, Tr H8, Layer 16, depth 4.2m, Field INM-I/ 1328. $\mathrm{NaOH}$ pretreatment.

\section{PRL-77. Chalcolithic culture}

$3310 \pm 110$

1360 вC

Charcoal, Loc H8, Layer 15, depth 3.2m, Field 1324. NaOH pretreatment.

PRL-76. Chalcolithic culture

$3220 \pm 110$ $1270 \mathrm{BC}$

Charcoal, Loc G8, Layer 7 , depth $.75 \mathrm{~m}$, Field 1094. NaOH pretreatment.

PRL-133. Chalcolithic culture

$3230 \pm 105$

$1280 \mathrm{BC}$

Charcoal, Loc INM-II, Layer 3, depth .3m, Field INM-II/1330. $\mathrm{NaOH}$ pretreatment.

PRL-78. Chalcolithic culture

$2740 \pm 115$ $790 \mathrm{BC}$

Charcoal, Loc H9-19, House 38 , sealed by Layer 7, depth $.75 \mathrm{~m}$, Field 1197. NäOH pretreatment.

PRL-93. Chalcolithic culture

$3020 \pm 105$

$1070 \mathrm{BC}$

Charcoal, Loc D7, House 36, Layer 4, Field 972. NaOH pretreatment.

PRL-94. Chalcolithic culture

$3020 \pm 115$ 1070 BC

Charcoal, Loc D8, Layer 4, Field 926. Rootlets removed, $\mathrm{NaOH}$ pretreatment. 


\section{PRL-22. Kanchipuram, India, early historic deposits $\begin{array}{cc}2360 \pm 120 & 410 \mathrm{BC}\end{array}$}

Charcoal from Kanchipuram (12。 $\left.50^{\prime} \mathrm{N}, 79^{\circ} 42^{\prime} \mathrm{E}\right)$, Dist Chingleput, Loc $\mathrm{O}^{\prime}-\mathrm{II}^{\prime}$, Layer 18 , depth $6.16 \mathrm{~m}$, Field KCM-1; subm by $\mathrm{T} V$ Mahalingam, Madras Univ, Madras.

\section{Khalaua series, Uttar Pradesh}

Khalaua $\left(27^{\circ} 6^{\prime} \mathrm{N}, 77^{\circ} 52^{\prime} \mathrm{E}\right)$, Dist Agra; subm by Dir Gen, Archaeol, New Delhi from PGW and Black and Red Ware (BRW) phase of early Iron age.

PRL-67. PGW and BRW deposits

Khalaua T2/66. Rootlets removed, $\mathrm{NaOH}$ pretreatment.

\section{PRL-68. PGW and BRW deposits}

Charcoal, Loc KHL-I, II and IV, Layer 8, depth 2.35 to $2.48 \mathrm{~m}$, Field Khalaua-T3/66. $\mathrm{NaOH}$ pretreatment.

PRL-142. Khangkhui, India, cave deposits

Modern

Charcoal from Khangkhui $\left(25^{\circ} 4^{\prime} \mathrm{N}, 94^{\circ} 25^{\prime} \mathrm{E}\right)$, Dist Manipur East, Tr 1, Layer 1 , depth $.2 \mathrm{~m}$, Field $\mathrm{KHK} / \mathrm{Cl}$; subm by $\mathrm{O} \mathrm{K}$ Singh, D M College, Imphal. $\mathrm{NaOH}$ pretreatment. Comment: date probably represents modern disturbance.

\section{Koldihawa series, Uttar Pradesh}

Koldihawa $\left(24^{\circ} 54^{\prime} 30^{\prime \prime} \mathrm{N}, 82^{\circ} 2^{\prime} 30^{\prime \prime} \mathrm{E}\right)$, Dist Allahabad; subm by G R Sharma, Allahabad Univ, Allahabad.

PRL-56. Chalcolithic culture (?)

$820 \pm 100$

Charcoal, Loc KDW-1, Al(a), Reference pit (c) sealed by Layer 1 , depth $.96 \mathrm{~m}$, Field AU/ALD/KDW/72/9. Rootlets removed.

\section{PRL-98. Chalcolithic culture (?)}

$1990 \pm 150$

Charcoal, Loc KDW-1, Al(b) III-VII, Layer 2, depth .23m, Field $\mathrm{AU} / \mathrm{ALD} / \mathrm{KDW} / 72 / 11$. Rootlets removed.

PRL-99. Chalcolithic culture

$$
2900 \pm 150
$$

Charcoal, Loc KDW-1, Al(a) O-III, Layer 4, depth .60m, Field $\mathrm{AU} / \mathrm{ALD} / \mathrm{KDW} / 72 / 12$. Rootlets removed.

PRL-100. Chalcolithic culture (?)

$7180 \pm 230$

Charcoal, Loc KDW-1, Al(a) O-III, Layer 5 , depth $.80 \mathrm{~m}$, Field $\mathrm{AU} / \mathrm{ALD} / \mathrm{KDW} / 72 / 13$. Rootlets removed. 
PRL-101. Chalcolithic culture (?)

$6300 \pm 180$

4350 вC

Charcoal, Loc KDW-1, Al(a), O-III, Layer 6, depth $1.1 \mathrm{~m}$, Field $\mathrm{AU} / \mathrm{ALD} / \mathrm{KDW} / 72 / 14$. Rootlets removed, $\mathrm{NaOH}$ pretreatment.

PRL-102. Chalcolithic culture

$2380 \pm 105$

430 BC

Charcoal, Loc KDW-1, Al(b), Ref Pit B sealed by Layer 1, depth $.42 \mathrm{~m}$, Field $\mathrm{AU} / \mathrm{ALD} / \mathrm{KDW} / 72 / 6$. $\mathrm{NaOH}$ pretreatment.

General Comment: wide scatter shows undetected disturbances at site.

PRL-46. Pallavamedu, India, Early historic deposit (?)

$17,480 \pm 500$

$15,530 \mathrm{BC}$

Charcoal from Pallavamedu $\left(12^{\circ} 50^{\prime} \mathrm{N}, 79^{\circ} 75^{\prime} \mathrm{E}\right)$, Dist Chingleput, Loc IV-V-IV'-V', Layer 5, depth 4m, Field PVM-1, subm by Dir Archaeol, Madras. $\mathrm{NaOH}$ pretreatment. Comment: sample probably mixed with lignite at site and has no relevance to cultural levels.

PRL-107. Patirajawela, Ceylon, coastal deposit

$4500 \pm 170$

2550 BC

Shells assoc with Mesolithic artifacts from Patirajawela $\left(6^{\circ} 10^{\prime} 20^{\prime \prime} \mathrm{N}\right.$, $81^{\circ} 13^{\prime} 30^{\prime \prime} \mathrm{E}$ ), Dist Hambantota, Loc D5, Layer II, depth .4m; subm by Dir Archaeol, Ceylon. Rootlets removed.

\section{Prabhas Patan series, Gujarat}

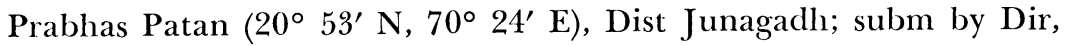
Deccan College, Poona.

PRL-19. Chalcolithic culture

$3100 \pm 160$

Charcoal, Loc PP-I Tr B6, Layer 9, depth $2.95 \mathrm{~m}$. NaOH pretreatment.

PRL-20. Chalcolithic culture

$3340 \pm 105$

1390 BC

Charcoal, Loc PP-I Tr C6, Layer 7, depth $2.12 \mathrm{~m}$. NaOH pretreatment.

PRL-90. Flood deposit

$4240 \pm 110$

2290 BC

Charcoal from a flood deposit, Loc D6, Layer 16, depth 6.5m, Field 506. $\mathrm{NaOH}$ pretreatment.

PRL-91. Chalcolithic culture

$3860 \pm 165$

$1910 \mathrm{BC}$

Charcoal from House 9, Loc C4, Layer 10, depth 3.3m, Field 421. $\mathrm{NaOH}$ pretreatment.

PRL-92. Chalcolithic culture

$3830 \pm 95$

$1880 \mathrm{BC}$

Charcoal from Tr D6-7, Layer 14, depth 4.85m, Field 495. NaOH pretreatment. 
PRL-97. Purana Qila, Delhi, early historic deposits

Charcoal from Purana Qila, Delhi, Loc PQL-N6 Qd 2, Layer 48, depth $10.35 \mathrm{~m}$, subm by Dir Gen, Archaeol, New Delhi. Rootlets removed.

\section{Sohgaura series, Uttar Pradesh}

Sohgaura $\left(26^{\circ} 23^{\prime} \mathrm{N}, 83^{\circ} 30^{\prime} \mathrm{E}\right)$, Dist Gorakhpur; subm by $\mathrm{S} \mathrm{N}$ Chaturvedi, Gorakhpur Univ, Gorakhpur. PRL-182 dates Iron age and PRL-179, -178 date Chalcolithic layers.

PRL-182a. NBP deposits

$2130 \pm 90$

Charcoal, Tr ZB2, Layer 9, depth 1.85m, sender's Sample 6a and b. $\mathrm{NaOH}$ pretreatment.

\section{PRL-179. BRW deposits}

$3090 \pm 130$

Charcoal, Tr ZB2, Layer 11, depth 3.02m, sender's Sample 3. NaOH pretreatment.

\section{PRL-178. BRW deposits}

$3190 \pm 110$

Charcoal, Tr ZB2, Layer 12, depth 3.15m, sender's Sample 2. $\mathrm{NaOH}$ pretreatment.

\section{PRL-85. Surkotada, India, Harappa culture}

$4140 \pm 130$

Charcoal from Surkotada $\left(23^{\circ} 37^{\prime} \mathrm{N}, 70^{\circ} 50^{\prime} \mathrm{E}\right)$, Dist Kutch, Loc ZF1, Qd 2, Layers 13 and 14, depth 4.85m, Field 21; subm by Dir Gen, Archaeol, New Delhi.

\section{Takiaper series, Uttar Pradesh} Varanasi.

Takiaper, Dist Varanasi; subm by $\mathrm{T}$ N Roy, Banaras Hindu Univ,

\section{PRL-184. NBP deposits}

$$
2070 \pm 100
$$

Charcoal, from burnt layer between deposits of Periods I and II, Layer 4, Field TKP-1. NaOH pretreatment.

\section{PRL-47. NBP deposits (?)}

$$
4600 \pm 120
$$

Charcoal, Loc Ao $2.58 \times .70$, Layer 5 , depth $1.2 \mathrm{~m}$. Rootlets removed, $\mathrm{NaOH}$ pretreatment. Comment: sample represents an earlier tree stump burnt at site.

PRL-61. Tilpat, India, wood of Adansonia digitata Modern

Wood pieces from Tilpat (28 $\left.30^{\prime} \mathrm{N}, 77^{\circ} 20^{\prime} \mathrm{E}\right)$, Dist Gurgaon. Coll by $\mathrm{K}$ M Vaid; subm by Vishnu Mittre, Birbal Sahni Inst Palaeobot, 
Lucknow. $\mathrm{NaOH}$ pretreatment. Comment: dated to validate reputed antiquity of wood.

\section{Togarappalli series, Tamil Nadu}

Togarappalli $\left(12^{\circ} 25^{\prime} \mathrm{N}, 78^{\circ} 22^{\prime} \mathrm{E}\right)$, Dist Dharmapuri. Coll by B Narasimhaiah; subm by M K Dhavalikar, Deccan College, Poona.

\section{PRL-134. Megalithic culture}

$2180 \pm 100$

Charcoal, Loc TGP-3, Layer 5, depth 1.05m, Field TGP-3. NaOH pretreatment.

\section{PRL-135. Megalithic culture}

$2150 \pm 110$

Charcoal, Loc TGP-3, Layer 5, depth 1.15m, Field TGP-3. NaOH pretreatment.

\section{REFERENCES}

Agrawal, D P, Gupta, S K, and Kusumgar, Sheela, 1971, Tata Institute date list IX: Radiocarbon, $\mathrm{v} 13, \mathrm{p} 442-449$. 